\title{
USO DE FIJADORES ESQUELÉTICOS EXTERNOS EN LA TÉCNICA DE DOBLE OSTEOTOMÍA PÉLVICA EN PERROS
}

\author{
Use of External Skeletal Fixators in the Double Pelvic Osteotomy \\ TeChNiQue In Dogs
}

\author{
José Panta V. ${ }^{1}$, Ysaac Chipayo G. ${ }^{1,2}$, Diego Díaz C. ${ }^{1}$, Víctor Fernández A. ${ }^{1}$
}

\section{Resumen}

El objetivo del presente estudio fue evaluar el uso de fijadores esqueléticos externos como medio de estabilización, mediante la técnica de doble osteotomía pélvica en pacientes displásicos. Se intervinieron cinco canes mestizos (3 machos y 2 hembras), de 3-4 meses de edad, con peso corporal entre 9.4 y $12.5 \mathrm{~kg}$, clínicamente sanos. El ángulo de Norberg se determinó radiológicamente antes de la intervención y al retiro de los implantes en la semana 6. Se les hizo un seguimiento radiológico postquirúrgico semanal por cinco semanas para determinar la formación del callo óseo. El 80\% (4/5) de los canes presentó signos radiológicos de formación de callo óseo a la segunda semana, y el 100\% (5/5) presentó un callo óseo muy desarrollado asociado a un adecuado aporte de estabilidad mecánica en la semana 5 postoperatoria. Todos los canes presentaron en promedio 9.4 grados de aumento del ángulo de Norberg (rango: 4 a 14º). Los resultados indican que la fijación esquelética externa ofrece buena estabilidad para el desarrollo de la doble osteotomía pélvica.

Palabras clave: displasia de cadera, fijadores esqueléticos externos, doble osteotomía pélvica, congruencia articular, ángulo de Norberg

\section{Abstract}

The aim of this study was to evaluate the use of external skeletal fixators as a means of stabilization, using the technique of double pelvic osteotomy in patients with hip dysplasia. Five crossbred dogs (3 males and 2 females), 3-4 months old, weighing 9.4 to $12.5 \mathrm{~kg}$, and clinically healthy were used. The Norberg angle was radiological assessed before and after 6 weeks of the surgery when the external fixators were removed. Weekly

\footnotetext{
${ }^{1}$ Clínica de Animales Menores, Facultad de Medicina Veterinaria, Universidad Nacional Mayor de San Marcos, Lima

${ }^{2}$ E-mail: ichipayo@hotmail.com
}

Recibido: 19 de abril de 2011

Aceptado para publicación: 24 de julio de 2013 
$\mathrm{X}$-rays for five weeks after the surgery were taken to monitor the development of the bone callus. The $80 \%$ (4/5) of dogs showed radiographic evidence of bone callus formation on week 2 and all dogs showed a well-developed bone callus associated with adequate contribution of mechanical stability on week 5. The Norberg angle increased 9.4 degrees on average (Range: 4 to 14 degress). The results showed that external skeletal fixation provides good stability when using the double pelvic osteotomy.

Key words: hyp displasia, external skeletal fixation, double pelvic osteotomy, joint congruity, Norberg angle

\section{INTRODUCCIÓN}

La displasia de cadera es una enfermedad ósea hereditaria producida por la malformación de la articulación coxofemoral, que genera diversos grados de inestabilidad articular y puede llegar a producir una enfermedad degenerativa articular crónica con dolor y cojera en el perro. Es de distribución mundial y afecta principalmente a canes de razas grandes y gigantes. El paciente nace con caderas normales y desarrolla la malformación durante los primeros meses de edad, iniciando con la laxitud de la cápsula articular, y con el tiempo ocurre la aparición de osteoartrosis (Hosgood et al., 1998).

El dolor que presenta el animal en casos avanzados de la enfermedad se maneja usualmente con antiinflamatorios, modificadores del líquido articular y corticoides. El reemplazo total de cadera no es una alternativa viable en el país, de allí que la mayoría de los pacientes son sometidos a cirugía radical de exéresis de cabeza y cuello del fémur, buscando eliminar la articulación, reduciendo el dolor y mejorando la calidad de vida del paciente.

Un diagnóstico precoz de displasia de cadera permite mejorar quirúrgicamente la congruencia articular, reduciendo drásticamente la probabilidad de presentación de osteoartrosis. Para esto, se dispone de exámenes clínicos y radiológicos como las pruebas de Ortolani, de Barlow, de Barden, y la medición de ángulo de Norberg, del índice de distracción y el de la proyección del borde dorsal del acetábulo (Dassier, 2006).
Se han desarrollado diversas técnicas quirúrgicas para reducir la presentación de osteoartrosis en los pacientes displásicos, pero con el tiempo han ido quedando en desuso debido a sus altos índices de fracaso y complicaciones postoperatorias. Una de las técnicas actuales utilizadas para el tratamiento de displasia de cadera es la doble osteotomía pélvica, la cual se realiza a edades tempranas (4 a 5 meses), pero requiere instrumental quirúrgico especial e implantes específicamente desarrollados para lograr un giro ventrolateral del acetábulo, que ayuda a mejorar la congruencia articular, evitando la presentación de la osteoartrosis (Hodsgood et al., 1998; Piermattei y Flo, 1999; Schulz y Dejardin, 2006).

Debido a la falta de implantes necesarios para el desarrollo de la técnica en el país, el presente estudio tuvo por objetivo el uso de los fijadores esqueléticos externos (FEE) para lograr estabilizar la osteotomía a nivel del íleon y permitir, a su vez, lograr la rotación del acetábulo mejorando la congruencia articular.

\section{Materiales y Métodos}

\section{Lugar del Estudio y Animales}

El estudio se realizó entre marzo y agosto de 2009 en la Clínica de Animales Menores de la Facultad de Medicina Veterinaria de la Universidad Nacional Mayor de San Marcos, Lima. Se utilizaron cinco canes (3 machos y 2 hembras) de raza mestiza, de 3 a 4 meses de edad y con 9.4 a $12.5 \mathrm{~kg}$ de peso 
vivo. Los animales estaban clínicamente sanos y con su calendario de vacunaciones y desparasitaciones al día.

\section{Evaluación Físico-Clínica y Radiológica}

Los canes fueron sedados con $15 \mathrm{mg} / \mathrm{kg}$ de ketamina y $0.25 \mathrm{mg} / \mathrm{kg}$ de diazepam, vía endovenosa, para la toma de placas radiográficas en posición ventro-dorsal, así como para medir el índice de distracción y de proyección del borde acetabular dorsal.

Se utilizó un transportador, regla y un vernier para determinar el ángulo de Norberg, el índice de distracción y el ángulo la proyección del borde acetabular dorsal.

\section{Técnica Quirúrgica}

Se realizó la Doble Osteotomía Pelvica (DPO), interviniéndose el lado de la cadera que presentaba menor ángulo de Norberg. El paciente fue preparado para abordaje de la zona lateral, caudal y ventral de la pelvis; y posicionado en decúbito lateral, con la articulación a ser operada hacia arriba. El miembro se rasuró desde el tarso hasta la línea media. El acto quirúrgico fue separado en tres tiempos:

\section{Ostectomía del pubis:}

Con el animal en decúbito lateral y la extremidad en abducción, se expuso la rama del pubis con un abordaje cutáneo ventral. La incisión de la piel se hizo sobre el borde craneal del músculo pectíneo, iniciando a la altura de la incisura acetabular. Se seccionó el músculo pectíneo cerca de su origen en la prominencia ileopúbica y tendón prepúbico. Se retrajo el músculo teniendo cuidado de proteger la arteria femoral, vena y nervio safeno. La rama acetabular del pubis se eliminó mediante el uso de la gubia sobre la prominencia ileopúbica y hasta la parte cercana al acetábulo. Este corte se hizo lo más cerca al cuerpo del íleon para minimizar la longitud del fragmento que se giró dentro del canal pélvico. Los músculos abdominales y el tendón prepúbico se suturaron al borde craneal del músculo gracilis y el resto de los tejidos se suturaron por capas.

\section{Corte del ligamento sacro tuberoso:}

Con el paciente en decúbito lateral, se procedió a reconocer el ligamento sacro tuberoso, el cual se inserta dorsal a la tuberosidad isquiática. Se realizó una incisión cutánea sobre el mismo ligamento, perpendicular a la línea media del cuerpo. Se disecó el ligamento cerca a su inserción en la tuberosidad isquiática y se seccionó. Se procedió al cierre de los tejidos por capas.

\section{Osteotomía del íleon:}

Se realizó el abordaje lateral mediante una incisión que se extendió desde el centro de la cresta iliaca y terminó justo caudal y distal del trocánter mayor. Se incidieron los tejidos subcutáneos, grasa glútea y fascia superficial; se realizó una incisión de la fascia glútea profunda a nivel de la incisión de piel. Se incidió el tabique intermuscular entre el tensor de la fascia lata y el músculo glúteo medio, complementando esta incisión a lo largo del borde craneal del bíceps femoral para formar una incisión fascial en forma de $\mathrm{T}$.

Para terminar la exposición del íleon, se continuó elevando los músculos glúteos del cuerpo y del ala ventral del íleon. La retracción del músculo glúteo medio expuso al músculo glúteo profundo y una parte del cuerpo iliaco. Se practicó una incisión en el origen del músculo glúteo medio sobre el íleon, empezando en la espina iliaca ventral caudal y se continuó craneal y dorsalmente, teniendo cuidado en proteger los nervios glúteos craneal, obturador e isquiático; este último ubicado cerca de la parte dorsomedial del cuerpo iliaco. Se levantaron todos los músculos ventral, medial y dorsalmente que cubrían la superficie lateral del cuerpo del íleon. También se realizó una incisión en el origen del músculo glúteo profundo para permitir su retracción caudal. 
Se practicó una osteotomía iliaca justo caudal respecto al sacro. Se realizó perpendicular en ambos planos respecto a una línea situada entre la cara dorsal de la tuberosidad isquiática y el tercio ventral de la cresta iliaca. El objetivo es hacer una osteotomía perpendicular al eje de rotación de la parte acetabular, en lugar de perpendicular al eje longitudinal del íleon. Para esto, se empleó un osteótomo y martillo, eliminando el borde dorsal saliente del fragmento caudal para evitar el daño sobre la musculatura glútea y el nervio isquiático.

Una vez realizada la osteotomía, se procedió a colocar los clavos de fijación esquelética externa basándose en los puntos descritos por Hickman (1964): tres en el segmento craneal y tres en el segmento caudal, estando el último en la tuberosidad isquiática. Al terminar la fijación de los clavos, se procedió al cierre del abordaje, suturando las fascias del músculo glúteo medio y el sartorio, y se continuó caudalmente entre el músculo glúteo medio y tensor de la fascia lata. La fascia glútea profunda, tejido subcutáneo y piel se fijaron por planos.

Posteriormente se hizo una rotación aproximada de $20^{\circ}$ del fragmento caudal a la osteotomía iliaca sujetándolo de sus tres clavos mediante una guía. Para colocar el metilmetacrilato previamente preparado, se deben sujetar los clavos hasta que la barra conectora brinde la estabilidad adecuada.

\section{Seguimiento Postoperatorio}

Se hizo un seguimiento con placas radiográficas en las semanas $1,2,3,4$ y 5 postquirúrgicas con los canes bajo sedación ligera en posición ventrodorsal y laterolateral para determinar la formación del callo óseo. Luego del retiro de los clavos, se procedió a determinar el ángulo de Norberg con el can bajo sedación mediante placa radiográfica ventrodorsal.

Se hizo una evaluación clínica para determinar el tiempo de inicio de la marcha, así como de posibles complicaciones clínicas.

\section{Resultados}

El 80\% (4/5) de los casos presentó los primeros signos radiológicos de formación de callo en la segunda semana postquirúrgica, y el 60\% (3/5) de los animales presentó un estado de condensación de callo óseo avanzado asociado con un adecuado aporte de estabilidad mecánica en la semana 4. Asimismo, todos los animales presentaron un callo óseo muy desarrollado asociado con un adecuado aporte de estabilidad mecánica en la semana 5 (Cuadro 1).

La variación del ángulo de Norberg por efecto de la cirugía se muestra en el Cuadro 2. La evolución del apoyo del miembro pélvico correspondiente a la cadera operada se muestra en el Cuadro 3. El 80\% (4/5) de los canes intervenidos tuvo un apoyo completo del miembro pélvico en el día 7, aunque uno de ellos tuvo ciertas dificultades para el apoyo completo del miembro hasta el día 13 postquirúrgico. El Cuadro 4 muestra las complicaciones observadas en cada uno de los canes intervenidos durante las 6 semanas de seguimiento.

\section{Discusión}

Los datos obtenidos en este estudio son los primeros en el país que fundamentan el uso de un sistema de fijación esquelética externa (FEE) para la realización de la técnica de Doble Osteotomía Pélvica que permite conservar los principios de rotación acetabular y estabilización de la osteotomía iliaca.

Se utilizó el esquema de Hickman (Hickman, 1964) para la colocación de fijadores esqueléticos externos en el caso de fracturas de íleon, y el metilmetacrilato como barra conectora, la cual ha sido descrita para superar la dificultad de alinear los implantes luego del giro ventrolateral del acetábulo (Piermattei y Flo, 1999; Egger, 2001). Fitzpatrick et al. (2008) evaluaron con éxito el uso de placas óseas lineales frente a FEE 
Cuadro 1. Evaluación radiológica de la evolución de la formación del callo óseo en pacientes con doble osteotomía pélvica tratados con fijación esquelética externa

\begin{tabular}{cccccc}
\hline \multirow{2}{*}{ Paciente } & \multicolumn{5}{c}{ Semana postoperatoria } \\
\cline { 2 - 6 } & 1 & 2 & 3 & 4 & 5 \\
\hline 1 & 0 & 0 & + & ++ & +++ \\
2 & 0 & + & ++ & +++ & +++ \\
3 & 0 & + & ++ & +++ & +++ \\
4 & 0 & + & ++ & ++ & +++ \\
5 & 0 & + & ++ & +++ & +++ \\
\hline
\end{tabular}

0 Márgenes fracturarios definidos, no se observa formación de callo óseo

+ Bordes fracturarios irregulares, inicio de mineralización del callo óseo

+ Callo óseo denso de tamaño reducido

+++ Condensación mayor del callo óseo

Cuadro 2. Variación del ángulo de Norberg por efecto de la cirugía medido a través de seguimiento radiológico en pacientes con doble osteotomía pélvica tratados con fijación esquelética externa

\begin{tabular}{|c|c|c|c|}
\hline \multirow{2}{*}{ Paciente } & \multicolumn{2}{|c|}{ Cirugía } & \multirow{2}{*}{ Variación } \\
\hline & Antes & Después ${ }^{1}$ & \\
\hline 1 & $107^{\circ}$ & $119^{\circ}$ & $12^{\circ}$ \\
\hline 2 & $109^{\circ}$ & $115^{\circ}$ & $6^{\circ}$ \\
\hline 3 & $104^{\circ}$ & $108^{\circ}$ & $4^{o}$ \\
\hline 4 & $93^{\circ}$ & $107^{\circ}$ & $14^{\circ}$ \\
\hline 5 & $100^{\circ}$ & $111^{\circ}$ & $11^{\circ}$ \\
\hline
\end{tabular}

${ }^{1}$ Seis semanas después de la cirugía

como medio de estabilización de fracturas no desplazadas a nivel del íleon, de allí que esos resultados fueron utilizados como base para el reemplazo de las placas de Slocum en este estudio.

El tiempo de formación de callo óseo fue menor a las ocho semanas reportado por Borostyankoi et al. (2003) en 95 canes de 10.8 meses de edad y del tiempo de 3-4 meses reportado por Altunatmaz et al. (2003) en 31 canes de 6 a 16 meses, para la curación de la osteotomía iliaca en la técnica de triple osteotomía pélvica donde usaron pla- cas de Slocum como medio de estabilización. Asimismo, el apoyo del miembro con capacidad de soporte de peso en el día 13 postquirúrgico en todos los canes fue menor a los 20-25 días reportado por Altunatmaz et al. (2003). El menor tiempo observado en el presente estudio podría deberse a la menor edad de los animales intervenidos.

El corto tiempo requerido para la recuperación de los canes demostraría que el sistema de FEE, además de adecuarse de manera maleable a la necesidad de la rotación ventrolateral de la ceja acetabular dorsal, 
Cuadro 3. Tiempo de apoyo total del miembro pélvico correspondiente a la cadera operada en pacientes con doble osteotomía pélvica tratados con fijación esquelética externa

\begin{tabular}{ccccccc}
\hline & & \multicolumn{7}{c}{ Día postoperatorio } \\
\cline { 2 - 7 } & Paciente & 1 & 4 & 7 & 10 & 13 \\
\hline & + & + & + & ++ & ++ & +++ \\
& + & ++ & +++ & ++ & +++ \\
& 3 & ++ & ++ & +++ & +++ & +++ \\
& 4 & ++ & ++ & +++ & +++ & +++ \\
& 5 & ++ & ++ & +++ & +++ & +++ \\
\hline 0 & No apoyo & & & & \\
+ & Marcada cojera de apoyo, por momentos mantiene miembro elevado \\
++ & Leve cojera de apoyo, por momentos mantiene peso de tren posterior con el miembro operado \\
+++ & Sin cojera, trote uniforme
\end{tabular}

Cuadro 4. Complicaciones postoperatorias en pacientes con doble osteotomía pélvica tratados con fijación esquelética externa

\begin{tabular}{|c|c|}
\hline Paciente & Complicación postoperatoria \\
\hline 1 & $\begin{array}{l}\text { Día 22: Se evidenció movimiento del clavo sobre la tuberosidad isquiática } \\
\text { durante la extensión de los miembros para control radiológico. }\end{array}$ \\
\hline 2 & $\begin{array}{l}\text { Día 9: Se encontró cojera de elevación. Respondió a tratamiento con } \\
\text { analgésico y antiinflamatorio. }\end{array}$ \\
\hline \multirow[t]{2}{*}{3} & $\begin{array}{l}\text { Día 2: Edema en zona de ostectomía púbica. Desapareció progresivamente al } \\
\text { día } 5 .\end{array}$ \\
\hline & Día 13: Se evidenció drenaje de líquido sanguinolento a nivel del $2^{\circ}$ clavo. \\
\hline 4 & No se observó complicaciones. \\
\hline \multirow[t]{3}{*}{5} & $\begin{array}{l}\text { Día 1: Edema en zona de ostectomía púbica. Desapareció progresivamente al } \\
\text { día } 3 .\end{array}$ \\
\hline & Día 6: Laceración de la piel, altura del primer clavo. \\
\hline & $\begin{array}{l}\text { Día 28: Presentó cojera de elevación. Respondió a antiinflamatorios y } \\
\text { analgésicos, recuperándose el día } 31 \text {. }\end{array}$ \\
\hline
\end{tabular}

aporta suficiente estabilidad durante el proceso de curación de la fractura para lograr la consolidación fracturaria no complicada de los fragmentos (San Román et al., 1998; Piermattei y Flo, 1999; Egger, 2001).

En el presente estudio se obtuvo 9.4 grados de aumento en promedio del ángulo de Norberg en los cinco pacientes con una rotación intraquirúrgica aparente de $20^{\circ}$ al cabo de seis semanas de seguimiento. De la Fuente (1995), trabajando con 15 caninos de 2 a 3 años de edad y con placas de Slocum de $45^{\circ}$ de rotación, encontró $15.5^{\circ}$ de variación entre las mediciones pre y postquirúrgica al cabo de 30 días, lo que permite sugerir que con 
rotaciones intraquirúrgicas mayores el grado de éxito en el aumento del angulo de Norberg al realizar la DPO con FEE también podría ser mayor, lo que permitiría el tratamiento en los pacientes con mayores deficiencias.

\section{Conclusiones}

- La fijación esquelética externa utilizada en la doble osteotomía pélvica brindó buena estabilidad a nivel de la osteotomía iliaca para la osteosíntesis del hueso.

- Los fijadores esqueléticos externos permitieron hacer el giro ventrolateral del acetábulo aumentando en todos los pacientes intervenidos el ángulo de Norberg.

- La fijación esquelética externa es una alternativa para estabilizar la osteotomía iliaca y permitir la formación de callo óseo.

\section{LitTeratura Citada}

1. Altunatmaz K, Yucel R, Devecioglu Y, Saroglu M, Ozsoy S. 2003. Treatment of canine hip displasia using triple pelvic osteotomy. Vet Med Czech 48: 41-46.

2. Borostyankoi F, Rooks RL, Kobluk CN, Reed AL, Littledike ET. 2003 Result of single-session bilateral triple pelvic osteotomy with an eight-hole iliac bone plate in dog: 95 cases (1996-1999). J Am Vet Med Assoc 222: 54-59.

3. Dassier C. 2006. Displasia de cadera en caninos: diagnóstico y tratamiento no quirúrgico. En: Slater D (ed). Tratado de cirugía en pequeños animales. $3^{\mathrm{a}}$ ed. Buenos Aires: Intermédica. p 2306-2315.

4. De la Fuente J. 1995. Hiperinclinación acetabular experimental en el perro. Tesis Doctoral. España: Univ Autónoma de Barcelona. $197 \mathrm{p}$

5. Egger EL. 2001. Fijación esquelética externa. En: Bojrab MJ, Gary WE, Barclay S (eds). Técnicas actuales en cirugía de pequeños animales. $4^{\circ}$ ed. Buenos Aires: Intermédica. p 876-884.

6. Fitzpatrick N, Lewis D, Cross A. 2008. A biomechanical comparison of external skeletal fixation and plating for the stabilization of ilial osteotomies in dogs. Vet Comp Orthop Traumatol 21:349-357.

7. Hickman J. 1964. Veterinary orthopaedics. Philadelphia: JB Lippincott. 468 p.

8. Hosgood G, Hoskins J, Davidson J, Smith J. 1998. Medicina y cirugía pediátrica de los animales de compañía. Zaragoza: Acribia. $361 \mathrm{p}$.

9. Piermattei D, Flo G. 1999. Manual de ortopedia y reparación de fracturas de pequeños animales. $3^{\mathrm{a}}$ ed. Madrid: McGraw-Hill Interamericana. 756 p.

10. San Román F, García P, Llorens P, Rodríguez J, Zaera J. 1998. Reducción y fijación de las fracturas. En: Sánchez-Valverde M (ed). Traumatología y ortopedia de pequeños animales. Madrid: McGraw-Hill. p 57-78.

11. Schulz K, Dejardin L. 2006. Tratamiento quirúrgico de la displasia de cadera en caninos. En: Slater D (ed). Tratado de cirugía en pequeños animales. $3^{\mathrm{a}} \mathrm{ed}$. Buenos Aires: Intermédica. p 2317-2349. 